\title{
CASO 2-2017: Mielinolisis pontina y extrapontina en una paciente con hiponatremia sintomática secundaria a uso de fármacos e hipopituitarismo postquirúrgico.
}

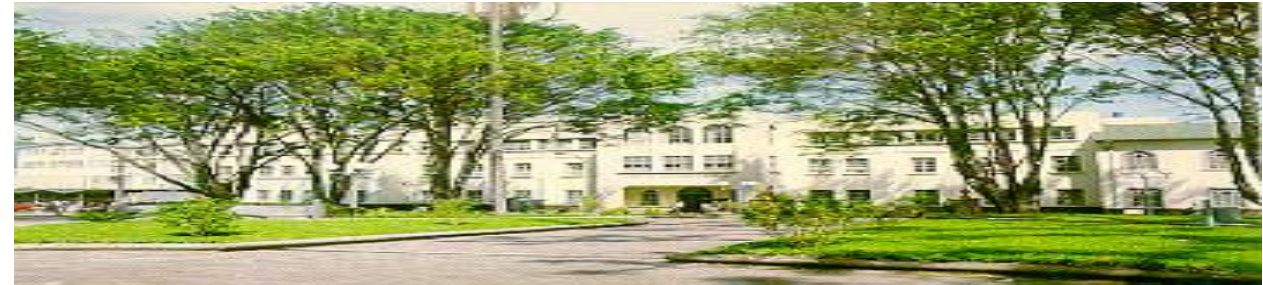

Hospital San quan de Dias. San José. Casta Rica. Fundado en 1845

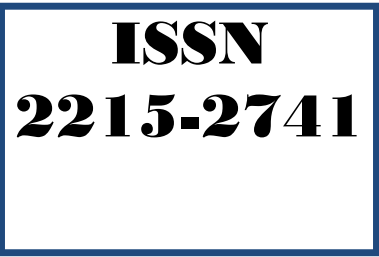

Recibido:

Aceptado:

/I

/I

Lucía Natalia Picado Leiva ${ }^{1}$

Alejandro Cob Guillen ${ }^{2}$

${ }^{1}$ Médica Residente de Neurología. PPEM UCR-CENDEISSS. Hospital San Juan de Dios. CCSS. Costa Rica. Correo electrónico: lucinatipicado@gmail.com

${ }^{2}$ Médico Residente de Endocrinología. PPEM UCR-CENDEISSS. Hospital San Juan de Dios. CCSS. Costa Rica.

\section{RESUMEN}

El síndrome de desmielinización osmótica (SDO) es una entidad que abarca la desmielinización central pontina (DCP) y la desmielinización extrapontina (DEP). Se caracteriza por daño a las vainas de mielina y a los tractos de sustancia blanca. La presentación clínica es heterogénea y el principal factor asociado al SDO es la hiponatremia; sin embargo se ha asociado también a diversas patologías. Se presenta el caso de una paciente con hipopituitarismo post-quirúrgico, quien asociado al uso de tiazidas, fluoxetina, carbamazepina y suspensión abrupta del uso crónico de esteroides, desarrolló hiponatremia sintomática. Asociado con la rápida corrección de ésta; presentó deterioro neurológico, con datos sugestivos de $\mathrm{SDO}$ en la resonancia magnética (RM).

\section{PALABRAS CLAVE}

Mielinolisis pontina y extrapontina. Hiponatremia sintomática. Tiazidas.

ABSTRACT 
Osmotic demyelination syndrome (ODS) refers to central pontine myelinolysis and extrapontine myelinolysis; this disorder is caused by injury of the myelin sheath and white matter tracts. Clinical manifestations are diverse, being the main factor associated hyponatremia; however, it has been associated to some other conditions. We present the case of a patient with hypopituitarism, who developed symptomatic hyponatremia secondary to the use of tiazides, fluoxetine, carbamazepine and abrupt suspension of steroid chronic use, sodium was quickly restored; resulting in ODS.

\section{KEY WORDS}

Pontine and extrapontine myelinolisis; symptomatic hyponatremia; tiazides.

\section{CASO CLÍNICO}

Femenina de 54 años, con antecedente de macroadenoma hipofisiario no funcionante resecado por craneotomía hace aproximadamente 25 años; con secuelas de hipopituitarismo postquirúrgico y tratamiento farmacológico de sustitución de tres ejes, además en tratamiento con fluoxetina y carbamazepina por epilepsia lesional. Sin otros antecedentes patológicos de importancia. Presentó cuadro de 15 días de evolución de síntomas inespecíficos caracterizados por astenia, adinamia, náuseas y malestar general; se documentó que la paciente había estado utilizando erróneamente hidrocloratiazida $25 \mathrm{mg}$ BID, en lugar de la dosis usual hidrocortisona 20mg BID, por lo que fue referida al centro hospitalario.

Ingresó al servicio de emergencias y en los laboratorios del ingreso se documentó hiponatremia en $106 \mathrm{mEq} / \mathrm{L}$ y calemia en $3.2 \mathrm{mEq} / \mathrm{L}$, con líquido extracelular disminuido. Se inició reposición aguda de sodio con solución fisiológica al $0.9 \%$, con una velocidad calculada según el déficit del sodio corporal total. Asociado a esto, se reinició la hidrocortisona $100 \mathrm{mg}$ IV BID y se suspendieron los fármacos: hidroclorotiazida, carbamazepina y fluoxetina.

La natremia control a las 5 horas del ingreso fue de $116 \mathrm{mEq} / \mathrm{L}$ (velocidad incremento $2 \mathrm{mEq} / \mathrm{h}$ ); a las 16 horas: $128 \mathrm{mEq} / \mathrm{L}$ (velocidad $1,4 \mathrm{mEq} / \mathrm{h}$ ), y a las 45 horas: $141 \mathrm{mEq} / \mathrm{L}$ (velocidad $0,7 \mathrm{mEq} / \mathrm{h}$ ).
Dos días posteriores al ingreso, la paciente presentaba mínima respuesta al estímulo doloroso, cuadriparesia espástica, hipertonía e hiperreflexia.

Se realizó tomografía computarizada de cerebro sin medio de contraste, que documentó como único hallazgo patológico una zona de encefalomalacia, la cual correlacionaba con su antecedente quirúrgico. La paciente persistió con aumento en la natremia, pese a tratamiento con solución glucosado al 5\%, hasta valores máximos de $161 \mathrm{mE} / \mathrm{L}$. Continuó con deterioro clínico, hasta comprometer la protección de vía aérea, por lo que requirió ventilación mecánica asistida. Se coordinó resonancia magnética (RM) la cual se realizó 48 horas tras inicio de síntomas.

El estudio inicial mostró como único hallazgo patológico la zona de encefalomalacia frontal derecha como secuela quirúrugica. La paciente evolucionó a cuadriparesia flácida, sin respuesta a estímulos sin sedación; asoció posteriormente crisis convulsivas, por lo que requirió tratamiento con ácido valproico. Se realizó RM control, 18 días tras inicio de síntomas neurológicos, en la cual se reportaron lesiones que se comportaban hiperintensas en las secuencias T2 y FLAIR e hipointensas en $\mathrm{T} 1$, localizadas en la sustancia blanca subcortical frontal bilateral, periventriculares frontales y parietales bilaterales, que medían entre 1 y $4 \mathrm{~mm}$, no producían efecto de masa ni se reforzaban tras la administración de contraste. Se observó además un aumento en la intensidad de señal en las secuencias FLAIR y T2 de las cabezas y cuerpos de núcleos caudados, putamen, globos pálidos bilaterales, así como cápsulas externas y extremas, con aumento en la intensidad de señal en las secuencias de FLAIR y T2 de la base y porción intermedia de la protuberancia y mesencéfalo, observando algunas zonas con comportamiento hiperintenso en las secuencias de difusión e isointenso en ADC, principalmente en la protuberancia; todos estos cambios sugestivos de SDO.

La paciente presentó leve mejoría clínica, con apertura ocular espontánea, fijación de la mirada al llamado, respuesta a estímulos físicos y escasa movilidad de miembros superiores, sin embargo por complicaciones infecciosas, evolucionó desfavorablemente y falleció. 
En el caso de esta paciente, se presentaron varios factores como causa de su hiponatremia: el uso de tiazidas, la interrupción abrupta de la hidrocortisona, asociado con el uso de carbamazepina y fluoxetina. Estos factores al ser revertidos, se han asociado a un mayor riesgo de sobrecorrección de la natremia utilizando solución salina, ya que al suspender dichos fármacos se corrige el síndrome de secreción inapropiada de hormona antidiurética (SIADH) iatrogénico y hay grandes pérdidas urinarias de agua, con el consecuente aumento rápido de la natremia.

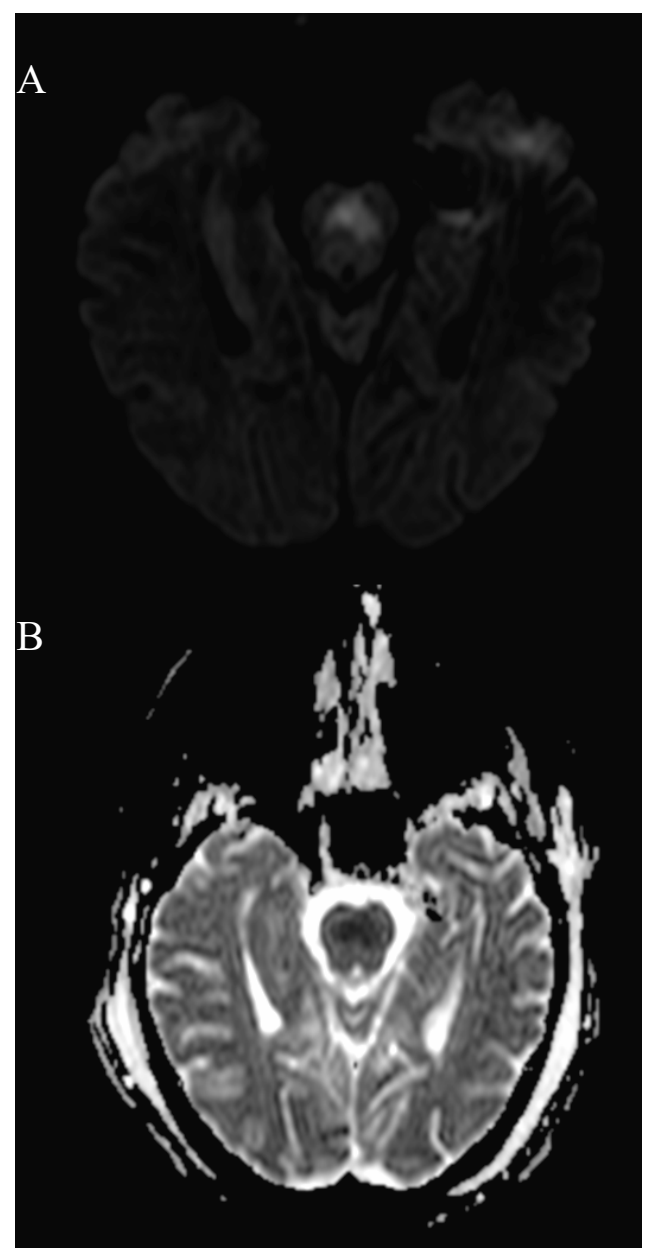

Figura 1. A: Secuencia DWI: restricción a la difusión en protuberancia. B: Secuencia ADC: lesiones isointensas en protuberancia.

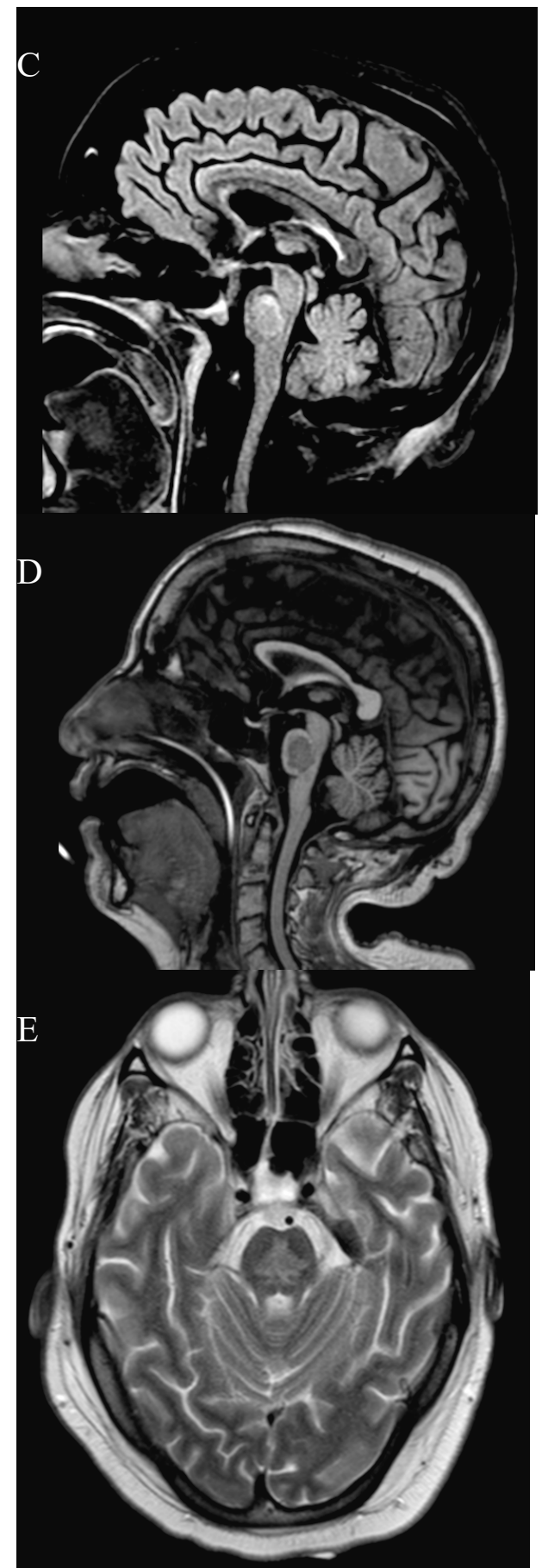

Figura 2. C: FLAIR sagital: Aumento de intensidad en protuberancia. D: T1 sagital: lesión hipointensa en protuberancia. E: T2 axial: lesiones hiperintensas en protuberancia en forma de "tridente" o "alas de murciélago". F: Espectroscopía de puente: aumento de pico colina, con una relación colina/creatina 2:1, presencia de lípidos y lactato. 


\section{DISCUSIÓN Y ANÁLISIS DEL CASO}

Introducción:

El SDO es un trastorno neurológico poco frecuente causado por daño a la vaina de mielina y lesión en tractos de sustancia blanca. El SDO abarca la DCP y DEP, siendo mucho más frecuente la DCP. El factor predisponente más común es la hiponatremia $(78 \%)$ y la presentación más común es la encefalopatía (30\%) según un metaanálisis realizado. La mitad de los pacientes tienen una recuperación favorable y la mortalidad ha ido disminuyendo. Inicialmente se documentó en pacientes alcohólicos y desnutridos; posteriormente se encontró asociación con ciertos trastornos electrolíticos y otras patologías agudas o crónicas.

En el $40 \%$ de los casos de DCP se encuentra DEP asociada, mientras que sólo el $11 \%$ de DEP se presenta de manera independiente a DCP.(1-3)

Presentación clínica:

La presentación clínica del SDO es bastante heterogénea; desde hallazgos incidentales asintomáticos o síntomas leves hasta coma o la muerte. Los pacientes con hiponatremia aguda ( $<48$ horas) comparados con los pacientes con hiponatremia crónica ( $>48$ horas) cursan por lo general con una presentación clínica más crítica; sin embargo, la hiponatremia que se desarrolla lentamente tiene mayor riesgo de DCP con la corrección de ésta. $(1,4)$

Clásicamente la DCP presenta un curso bifásico, por lo general, inicia con encefalopatía que empeora gradualmente seguido de deterioro neurológico severo manifestado por: disartria y disfagia (tractos corticobulbares), cuadriparesia flácida (tracto corticoespinal) y posteriormente cuadriparesia espástica (base de puente); cuando hay lesión mesencefálica también, puede haber disfunción pupilar y oculomotora; se ha descrito el "síndrome de cautiverio" en los casos más severos, con preservación únicamente de los movimientos de ojos verticales y parpadeo. $(1,3,4)$

En la DEP se puede encontrar: trastornos de comportamiento, trastornos psiquiátricos, trastornos de movimiento (parkinsonismo, distonía, tremor), crisis convulsivas, depresión, polirradiculopatía y neuropatía.(1)

Factores asociados:

El factor más asociado era la hiponatremia; siendo importantes la cronicidad y velocidad de correción del trastorno electrolítico para el desarrollo de la desmielinización; más que la concentración de sodio propiamente. $\mathrm{La}$ hiponatremia se ha asociado al uso de ciertos fármacos como: carbamazepina, inhibidores selectivos de la recaptura de serotonina (ej. fluoxetina), litio, antidepresivos tricíclicos, opioides, diuréticos (ej. tiazidas), AINES, entre otros.

También se ha asociado a diversas condiciones como son: síndrome de secreción inapropiada de hormona antidiurética, falla renal, diálisis, hepatitis, hiperemesis (gravídica, bulimia o por efecto farmacológico) y quemaduras. Además de la hiponatremia, el SDO se ha asociado a disfunción hepática, transplante de hígado, estados hiperosmolares, otros trastornos electrolíticos (hipofosfatemia, hipokalemia severa), diabetes mellitus, leucemia, linfoma, enfermedad de Wilson, embarazo, lupus eritematoso sistémico, patologías psiquiátricas (esquizofreina, anorexia, polidipsia psicogénica y abuso de éxtasis), entre otros.(1,3-5)

Fisiopatología:

No se conocen con exactitud los mecanismos fisiopatológicos del SDO, pero se cree que la DCP y DEP comparten una misma fisiopatología. El SDO se caracteriza por pérdida simétrica de mielina no inflamatoria con preservación de los cuerpos celulares y axones del puente; con preservación de fibras periféricas y los axones del tracto corticoespinal. En el SDO se encuentra disrupción de la barrera hematoencefálica (BHE) y las uniones endoteliales por estrés osmótico; resultando en edema vasogénico, compresión de tractos y mielinolisis, esto ya que ésta disrupción permite acceso de sustancias nocivas (complemento) a la mielina; además que las células endoteliales dañadas liberan otras sustancias nocivas como activador de plasminógeno y citoquinas. También hay pérdida de oligodendrocitos con 
preservación de neuronas y axones así como infiltración de macrófagos; esto en contraste con el componente inflamatorio de otras condiciones desmielinizantes como la Esclerosis Múltiple. $(1,3,4)$

Norenberg señala que en el caso de hiponatremia aguda; el agua se mueve de la sangre al cerebro, las células se hinchan (principalmente los astrocitos), hay liberación de iones inorgánicos (sodio, potasio y cloruro) para reestablacer el volumen celular normal; en contraste con la hiponatremia crónica donde hay pérdida de osmolitos orgánicos (mioinositol, taurina, glutamina, glutamato, creatina, fosfocreatina, glicerofosforilclorina); los iones inorgánicos retornan rápidamente al espacio intracelular, en contraste con los osmolitos orgánicos que se mueven más lentamente, lo que produce disfunción proteica durante mayor tiempo, por lo tanto la hiponatremia crónica debe ser corregida más lentamente para darle tiempo a las células cerebrales a adquirir nuevamente osmolitos orgánicos y electrolitos; ya que la corrección sérica rápida de sodio se asocia a un sobredisparo relativo del sodio cerebral y niveles de cloruro en la presencia de una concentración baja de osmolitos orgánicos, resultando en SDO. $(2,4)$

\section{Localización:}

La susceptibilidad del puente a ésta entidad puede ser porque hay sustancia blanca y gris mezclada, alta concentración de oligodendrocitos ya que están expuestos a una sustancia mielotóxica como resultado del estrés osmótico. Encontramos características similares en los sitios donde más se encuentra DEP: cerebelo, cuerpo geniculado lateral, cápsula externa y extrema, ganglios basales, tálamo, unión sustancia blanca-gris y el hipocampo. Se han descrito esporádicamente lesiones en médula espinal, cuerpos mamilares, columnas del fórnix, amígdala, comisura anterior, tractos ópticos, núcleo subtalámico. $(1,4)$

\section{Patología:}

Las características neuropatológicas de DCP son: lesiones simétricas, con bordes regulares en el centro del puente, con destrucción selectiva de vainas de mielina y pérdida de oligodendrocitos; macrófagos llenos de grasa, en ausencia de infiltrado inflamatorio. Los vasos sanguíneos están permeables, los axones y los cuerpos celulares por lo general están preservados. Las vainas de mielina se encuentran hinchadas y la mielina fragmentada. Se encuentran astrocitos hipertróficos localizados centralmente marcados con proteína acídica fibrilar glial (GFAP). En lesiones más antiguas, puede haber un área central de cavitación por degeneración de los elementos. Los hallazgos patológicos de la DEP son iguales a los de DCP.(4)

Imágenes:

Conforme ha aumentado el uso de la RM, se ha logrado identificar con mayor frecuencia el SDO. Con la secuencia imagen de difusión ponderada (DWI) se pueden encontrar anormalidades más tempranamente y con mayor sensibilidad; en el SDO se encuentra restricción a la difusión y un menor valor de coeficiente de difusión en coeficiente de difusión aparente (ADC) en el sitio de lesión. Las lesiones se ven hiperintensas en $\mathrm{T} 2$ y con atenuación de fluido por recuperación inversa (FLAIR) e hipointensas en $\mathrm{T} 1$, sin efecto de masa.

En el puente central se ven lesiones con forma de "tridente" o de "alas de murciélago". En la RM se pueden encontrar cambios desde las primeras 24 horas de inicio de los síntomas con retorno de los valores de base de la secuencia ADC en 3-4 semanas.; sin embargo, la aparición de cambio de señal en T2 puede atrasarse hasta 14-21 días tras inicio de sintomatología, por lo que se puede requerir repetición de RM. Graff-Radford et al reportaron que en $23 \%$ de sus pacientes, la RM inicial no mostraba evidencia de señal pontina, $\mathrm{y}$ en la segunda imagen, todos tenían evidencia de anormalidades. Por lo tanto, las imágenes seriadas son útiles en los casos de alta sospecha clínica que no muestren alteraciones en la RM inicial. Las lesiones en DCP normalmente no captan medio de contraste; únicamente lo hace un quinto de los casos. Con el tiempo, la anormalidad en DWI revierte y la señal de T2 resuelve completamente, aunque pueden haber zonas de gliosis residuales. $(1,3,5)$

En DEP se encuentra anormalidades simétricas en T2; principalmente en cerebelo (33\%), cuerpo geniculado lateral, en cápsula externa, extrema, ganglios basales, tálamo, unión sustancia blancagris e hipocampo.(1) La espectroscopía muestra 
niveles de colina bajos, aumento del radio colina/creatina, niveles de $\mathrm{N}$-acetilaspartato bajos y niveles lipídicos bajos. $(1,4)$

La tomografía computarizada (TC) es relativamente insensible; lo que se encuentra es hipodensidad en los sitios afectados.(1)

Tratamiento:

La prevención es el mejor tratamiento. La corrección de la hiponatremia no debe exceder $0.5 \mathrm{mmol} / \mathrm{hr}$ o $0.5 \mathrm{mEq} / \mathrm{hr}$; existe la fórmula de Adrogué-Madias para predecir el cambio en los niveles de sodio sérico en respuesta a líquidos intravenosos. La plasmaféresis y administración de inmunoglobulina intravenosa se han asociado a mejor pronóstico.(1.4,7)

Otros tratamientos potenciales: esteroides, tratamientos específicos para síntomas neurológicos y psiquiátricos (ej. agentes antiparkinsonianos para trastornos de movimiento o antipsicóticos para agitación), psicoterapia, entre otros. El tratamiento de soporte y de enfermedades concomitantes agresivo es vital. $(3,4)$

Pronóstico:

Según un metaanálisis, $51.9 \%$ de los pacientes tenían una recuperación favorable (un cuarto de estos con buena recuperación funcional) y $24.8 \%$ de los pacientes morían. Kallakatta et al. reportaron que hiponatremia $<115 \mathrm{mEq}$, con hipocalemia asociada y bajo puntaje en la GCS inicial se asociaban a un pobre pronóstico; mientras que entre mayor puntaje de GCS, mejor puntación en escalas funcionales, hiponatremia menos severa y ausencia de hipocalemia asociada predecían un mejor pronóstico. $(3,6)$

Los pacientes con SDO tienen mayor riesgo de complicaciones que ponen en peligro la vida como neumonía por aspiración, trombosis venosa profunda y embolismo pulmonar.(1)

Las características radiológicas como localización de la lesión, volumen y distribución de la lesión, restricción a la difusión y captación de medio de contraste en RM no influyen en el pronóstico clínico. La mortalidad ha ido disminuyendo por métodos diagnósticos más avanzados, mayor reconocimiento de fisiopatología y causantes del SDO y mejor tratamiento en la unidad de cuidados intensivos. $(3,5)$

\section{CONCLUSIONES}

Se ha documentado ciertos factores que empeoran el pronóstico de SDO: hiponatremia $<115 \mathrm{mEq}$, hipokalemia asociada y bajo puntaje en la escala GCS inicial. Con el uso de la RM se ha incrementado el diagnóstico y tratamiento temprano, mejorando así el pronóstico.

\section{BIBLIOGRAFÍA}

1. Alleman A. Osmotic Demyelination Syndrome: Central Pontine Myelinolysis and Extrapontine Myelinolysis. Semin Ultrasound CT MRI, 2014; 35:153-159

2. Norenberg MD. Central pontine myelinolysis: historical and mechanistic considerations Metab Brain Dis. 2010;25(1):97-106.

3. Singh $\mathrm{T}$ Fugate $\mathrm{J}$ Rabinstein A. Central pontine and extrapontine myelinolysis: a systematic review. European Journal of Neurology. 2014.;21:1443-1450.

4. Hurley R Filley C Taber K. Central pontine myelinolysis: a metabolic disorder of myelin, J Neuropsychiatry Clin Neurosci. 2011;23(4):369-374.

5. Graff-Radford J Fugate J Kaufmann T et al. Clinical and Radiologic Correlations of Central Pontine Myelinolysis Syndrome, Mayo Clin Proc. 2011;86(11):1063-1067.

6. Kallakatta R Radhakrishnan A Fayaz R et al. Clinical and functional outcome and factors predicting prognosis in osmotic demyelination syndrome (central pontine and extrapontine myelinolysis) in 25 patients. J Neurol Neurosurg Psychiatry. 2011;82(3):326-331.

7. Mohmand HK Issa D Ahmad $Z$ et al. Hypertonic saline for hyponatremia: risk of inadvertent overcorrection. Clin J Am Soc Nephrol. November 2007;2(6):1110-1117.

$\begin{array}{llll}\text { CONFLICTO DE } & \text { INTERÉS } & \text { Y/O } \\ \text { AGRADECIMIENTOS } & \end{array}$


Los autores declaran que no existió ningún conflicto de interés en el presente reporte. 